\title{
Simultaneous Determination of Paeoniflorin and Glycyrrhizin in Sayuk-san by HPLC/DAD
}

\author{
Mi Kyeong Lee', Ki Yong Lee², Seung Hyun Kim³, Min-Ji Jeon², Jung Hee Cho ${ }^{4}$, \\ Mi Hyun $\mathrm{Oh}^{4}$, Ju Hyun Baek ${ }^{4}$, Hyo Jin Kim ${ }^{5}$ and Sang Hyun Sung ${ }^{2 \dagger}$ \\ ${ }^{1}$ College of Pharmacy, Chungbuk National University, Cheongju 361-763, Korea \\ ${ }^{2}$ College of Pharmacy, Seoul National University, Seoul 151-742, Korea \\ 3 Institute for Life Science, Elcom Science Co.Ltd., Seoul 151-742, Korea \\ ${ }^{4}$ Herbal Medicine Evaluation Department, Korea Drug \& Food Administration, Seoul 122-704, Korea \\ ${ }^{5}$ College of Pharmacy, Dongduk Women's University, Seoul 136-714, Korea \\ (Received December 2, $2008 \cdot$ Revised January 19, $2008 \cdot$ Accepted February 9, 2008)
}

\begin{abstract}
A high performance liquid chromatographic (HPLC) method for the simultaneous determination of marker constituents, paeoniflorin and glycyrrhizin was established for the quality control of traditional herbal medicinal preparation, Sayuk-san (SYS). Separation and quantification were successfully achieved with a Waters XTerra RP18 column (5 $\mu \mathrm{m}$, $4.6 \mathrm{~mm}$ I.D. $\times 150 \mathrm{~mm}$ ) by gradient elution of a mixture of acetonitrile and water containing $0.03 \%$ phosphoric acid $(\mathrm{pH} 2.03)$ at a flow rate of $1.0 \mathrm{~mL} / \mathrm{min}$. The diode-array UV/vis detector (DAD) was used for the detection and the wavelength for quantification was set at $230 \mathrm{~nm}$. The presence of paeoniflorin and glycyrrhizin in this decoction was ascertained by retention time, spiking with each authentic standard and UV spectrum. All two compounds showed good linearity ( $\mathrm{r}^{2}$ $>0.996$ ) in a relatively wide concentration ranges. The R.S.D. for intra-day and inter-day precision was less than $7.3 \%$ and the limits of detection (LOD) were less than $55.7 \mathrm{ng}$. The mean recovery of each compound was $102.3 \sim 111.1 \%$ with R.S.D. values less than $4.6 \%$. This method was successfully applied to the determination of contents of paeoniflorin and glycyrrhizin in three commercial products of SYS. These results suggest that the developed HPLC method is simple, effective and could be readily utilized as a quality control method for commercial SYS products.
\end{abstract}

Key words - Sayuk-san (SYS), HPLC, Quantification, Validation

Traditional herbal medicine has been used for a long time in Asia including Korea and has been verified their therapeutic effects. Sayuk-san (SYS) is combinational herbal decoction comprising Ponciri Fructus, Bupleuri Radix, Glycyrrhizae Radix and Paeoniae Radix. SYS has been used in traditional medicine to relieve the symptoms of digestive disorders. In addition, SYS has been commercially produced as granules by several medicinal manufacturers in Korea.

As it is well known, traditional herbal medicinal preparations are mostly used in combination of many herbs. Multiple constituents from each herb are known to be responsible for their therapeutic effects, ${ }^{1)}$ however, the quality of each herb has been affected by many factors such as cultivation environment and manufacturing process. ${ }^{2-5}$ Moreover, even though each herb has been mixed in the same ratio, different preparation procedure such as cutting size of herbs, temperature, time, pressure for extraction may affect the amounts of various constituents in the decoction. In other words, all these factors can

†본 논문에 관한 문의는 이 저자에게로

Tel : 02)880-7859, E-mail : shsung@snu.ac.kr affect the therapeutic effects and/or safety of traditional medicinal preparation. Therefore, the need for quality assessment of major active components in traditional herbal medicinal preparation has been increased. As such, numerous studies related to quality control have been carried out, mainly by the determination of major and/or active constituents. ${ }^{6-9)}$

Several analytical methods have been reported for each herbal component of SYS; Glycyrrhizae Radix ${ }^{10-11)}$ and Paeoniae Radix. ${ }^{12)}$ However, there have been no reports about the simultaneous quantitative determination of their major constituents of SYS.

In the present study, the simultaneous quantitative analytical method for the marker constituents of SYS, paeoniflorin from Paeoniae Radix and glycyrrhizin from Glycyrrhizae Radix (Figure 1), was developed using HPLC/DAD and validated. In addition, the content of the marker constituents in three commercial SYS products was measured using this method.

\section{Experimental}

\section{Materials}

The marker compounds, paeoniflorin and glycyrrhizin, were 

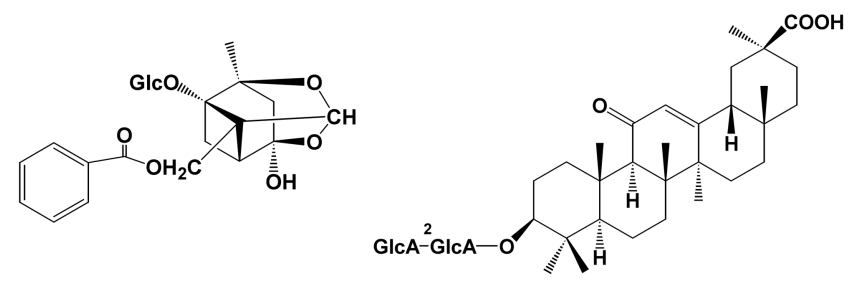

paeoniflorin

glycyrrhizin

Figure 1-Structures of four marker constituents of SYS.

purchased from Wako (Osaka, Japan). All of the plants were purchased from Kyungdong traditional herbal market (Seoul, Korea) and were authenticated by Prof. J.H. Park in the College of Pharmacy, Pusan National University. The commercial SYS products from medicinal companies were purchased from local providers. HPLC grade solvents (acetonitrile, water and methanol) and reagents were obtained from BDH chemicals (Poole, UK). Phosphoric acid (analytical grade) was purchased from Merck (Darmstadt, Germany). Triple distilled water (Millipore, Bedford, MA, USA) was used for all preparations.

\section{Chromatographic conditions}

The HPLC system was consisted of a chromatographic pump (P680, Dionex, Germany), an injector (7725i, Rheodyne, USA) equipped with Photo Diode Array (UVD 340U, Dionex, Germany). The output signal of the detector was recorded using a Dionex Chromelon ${ }^{\mathrm{TM}}$ Chromatography Data System. Chromatographic separation was achieved on a Waters XTerra ${ }^{\mathrm{TM}} \mathrm{C} 18(5 \mu \mathrm{m}, 4.6 \mathrm{~mm}$ I.D. $\times 150 \mathrm{~mm})$ by gradient elution of a mixture of acetonitrile and water containing $0.03 \%$ phosphoric acid $(\mathrm{pH} 2.03)$ at a flow rate of $1.0 \mathrm{~mL} / \mathrm{min}$. The diode-array UV/vis detector (DAD) was used for the detection and the wavelength for quantification was set at $230 \mathrm{~nm}$

\section{Preparation of standard solution}

Stock standard solution of the marker compounds was prepared in methanol at a concentration of $1 \mathrm{mg} / \mathrm{mL}$, respectively. The appropriate amount of every standard solution was mixed and diluted as indicated.

\section{Sample preparation for HPLC}

For the preparation of SYS sample for HPLC experiment, $2.0 \mathrm{~g}$ of Ponciri Fructus, $2.0 \mathrm{~g}$ of Bupleuri Radix, $1.0 \mathrm{~g}$ of Glycyrrhizae Radix and $2.0 \mathrm{~g}$ of Paeoniae Radix, were weighed accurately and mixed. Ten times of water $(90 \mathrm{~mL})$ was added to mixed herbs for SYS and refluxed for $2 \mathrm{~h}$ at $90^{\circ} \mathrm{C}$. The extract was filtered and evaporated in vacuo, and then sus- pended to $100 \mathrm{~mL}$ of $50 \%$ methanol. For the preparation of HPLC samples of commercial SYS products, dried commercial SYS granule samples were weighed accurately and extracted with $90 \%$ methanol for $1 \mathrm{~h}$ at $90^{\circ} \mathrm{C}$, using a reflux. The extraction was repeated three times. All the extract was filtered and evaporated in vacuo, and then suspended to $10 \mathrm{~mL}$ of $50 \%$ methanol. This sample solution was filtered through $0.45 \mu \mathrm{m}$ membrane filter (Millipore, Nylon, $170 \mathrm{~mm}$ ) and analyzed with HPLC.

\section{Results and Discussion}

\section{Chromatographic conditions}

For the simultaneous determination of the marker component of SYS, paeoniflorin and glycyrrhizin, the chromatographic condition was first investigated. Various mixtures of water and acetonitrile in combination with phosphoric acid were tested as a mobile phase. Acid is known to achieve better separation for organic compounds by depressing the tailing of the peaks. ${ }^{13,14)}$ In our chromatographic condition, addition of phosphoric acid in water increased the resolution of the peaks. The wavelength for detection was tested at 210, 230, 250 and $280 \mathrm{~nm}$ and set at $230 \mathrm{~nm}$, where the four compounds showed the maximum absorption as measured by a DAD detector. The presence of paeoniflorin and glycyrrhizin in this decoction was verified by comparing each retention time and UV spectrum with those of each standard compound, and spiking with authentic standards. As a result, the optimal gradient mobile phase consisting of acetonitrile-water with $0.03 \%$ phosphoric acid was subsequently employed for the analysis of SYS (Table 1), which led to good resolution and satisfactory peak shape at $230 \mathrm{~nm}$ (Figure 2B).

\section{Validation of the HPLC Methods}

Specificity was determined by the calculation of peak purity facilitated by the photodiode array detector (PDA) and the corresponding computer software. The absorption spectrum of a

Table 1-Solvent Gradient Conditions for HPLC-DAD

\begin{tabular}{cccc}
\hline \hline $\begin{array}{c}\text { Final time } \\
(\mathrm{min})\end{array}$ & Solvent $\mathrm{A}^{\mathrm{a}}$ & Solvent $\mathrm{B}^{\mathrm{b}}$ & $\begin{array}{c}\text { Flow rate } \\
(\mathrm{mL} / \mathrm{min})\end{array}$ \\
\hline 0 & 20 & 80 & 1.0 \\
13 & 40 & 60 & 1.0 \\
20 & 75 & 25 & 1.0 \\
23 & 20 & 80 & 1.0 \\
25 & 20 & 80 & 1.0 \\
\hline
\end{tabular}

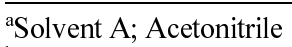

${ }^{\mathrm{b}}$ Solvent B; $0.03 \%$ phosphoric acid (pH 2.03) 
(A)

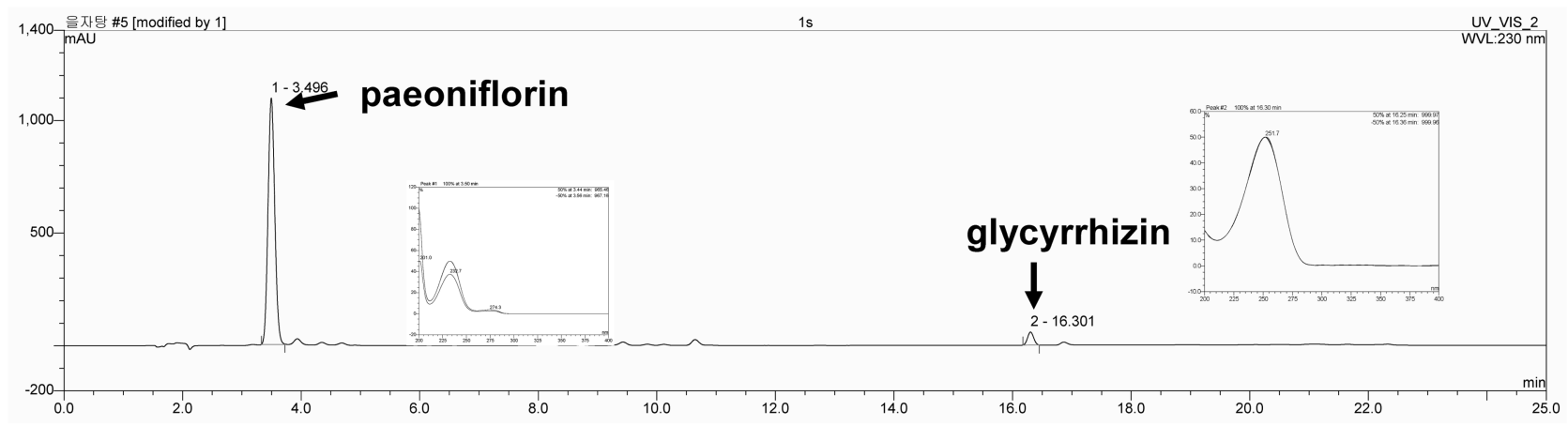

(B)

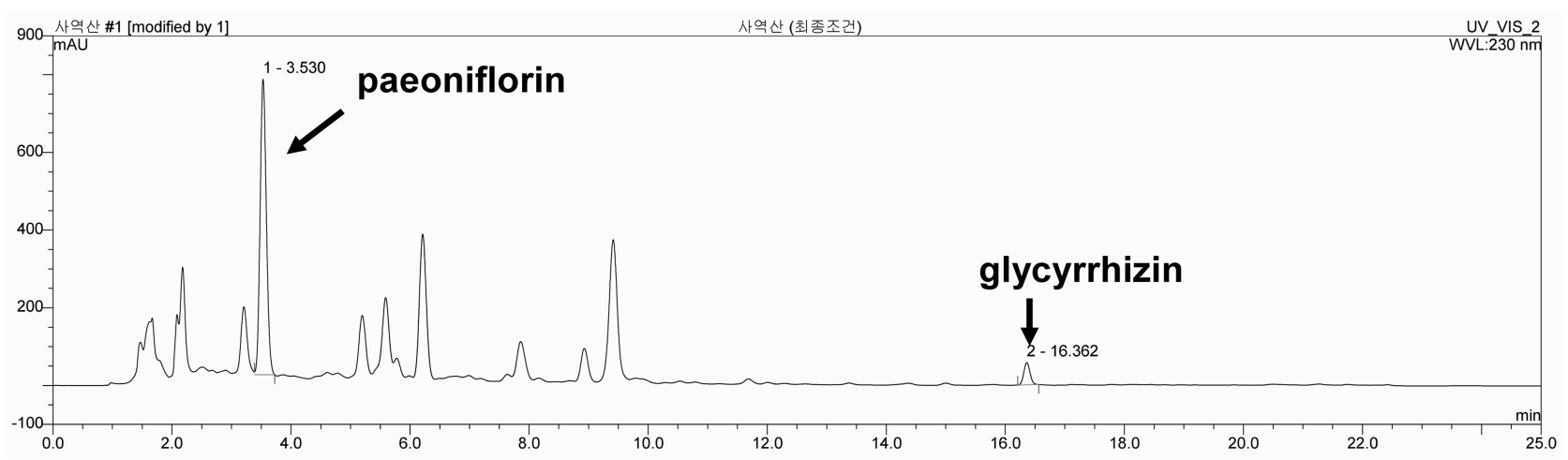

Figure 2-HPLC chromatogram of standard mixtures (A) and SYS (B).

Table 2-Linear Ranges, Limit of Detection (LOD) and Characteristic Parameters of Calibration Curves of Marker Constituents of SYS

\begin{tabular}{ccccccc}
\hline \hline \multirow{2}{*}{ Compound } & \multirow{2}{*}{$\begin{array}{c}\text { Linear range } \\
(\mu \mathrm{g} / \mathrm{mL})\end{array}$} & \multicolumn{2}{c}{ Linear regression equation ${ }^{\mathrm{a}} y=\mathrm{a} x+\mathrm{b}$} & & $\begin{array}{c}\text { Correlation coefficient } \\
\left(\mathrm{r}^{2}\right)\end{array}$ & $\begin{array}{c}\text { LOD } \\
(\mathrm{ng})\end{array}$ \\
\cline { 3 - 6 } & $2-400$ & Slope $(\mathrm{a})$ & Intercept $(\mathrm{b})$ & 0.1835 & 0.9977 & 55.7 \\
paeoniflorin & $1-200$ & 35.8750 & 0.0184 & 0.9960 & 24.1 \\
glycyrrhizin & 3.4341 & & & \\
\hline
\end{tabular}

a $y=$ peak area, $x=$ concentration $(\mu \mathrm{g} / \mathrm{mL})$

single component remained little variable at each time point in one peak, which supported specificity of each peak (Figure 2B). Our results clearly showed the specificity of each peak for paeoniflorin and glycyrrhizin, respectively.

The linearity of paeoniflorin and glycyrrhizin was calculated by seven concentrations of each compound. The regression equation and correlation coefficients $\left(r^{2}\right)$ were listed in Table 2 and high correlation coefficient values $\left(r^{2}>0.996\right)$ showed good linearity in relatively wide range of concentration.

Limit of detection (LOD) was measured based on the method recommended by $\mathrm{ICH}(\mathrm{LOD}=3.3 \delta / S, \delta=$ standard deviation of the response, $S=$ slope of the calibration curve).
LOD of paeoniflorin and glycyrrhizin were 55.7 and $24.1 \mathrm{ng}$, respectively, which showed a high sensitivity at this chromatographic condition.

The precision test was carried out by the intra-day and interday variability for these constituents. The intra-day variability was assayed at three concentrations on the same day and interday variability was assayed at three concentrations on three sequential days (1, 3, 5 days). As listed in Table 3, the mean accuracy of each compound was 91.9 104.4\%. The R.S.D. of intra-day and inter-day variability was less than $7.3 \%$, which demonstrated good precision of this method.

The recovery test was determined by the method of standard 
Table 3-Analytical Results of Intra-day and Inter-day Variability

\begin{tabular}{|c|c|c|c|c|c|c|c|}
\hline \multirow{2}{*}{ Compound } & \multirow{2}{*}{ Amount $(\mu \mathrm{g})$} & \multicolumn{3}{|c|}{ Intra-day } & \multicolumn{3}{|c|}{ Inter-day } \\
\hline & & Detected $(\mu \mathrm{g})$ & R.S.D. (\%) & Accuracy (\%) & Detected $(\mu \mathrm{g})$ & R.S.D. (\%) & Accuracy (\%) \\
\hline \multirow{3}{*}{ paeoniflorin } & 2.00 & $2.05 \pm 0.01$ & 0.52 & 92.4 & $2.06 \pm 0.09$ & 4.17 & 97.1 \\
\hline & 0.80 & $0.83 \pm 0.01$ & 0.28 & 96.0 & $0.78 \pm 0.06$ & 7.33 & 102.5 \\
\hline & 0.20 & $0.22 \pm 0.003$ & 1.52 & 91.9 & $0.21 \pm 0.02$ & 7.58 & 92.9 \\
\hline \multirow{3}{*}{ glycyrrhizin } & 1.00 & $1.00 \pm 0.02$ & 1.94 & 99.9 & $1.06 \pm 0.01$ & 1.10 & 93.7 \\
\hline & 0.40 & $0.41 \pm 0.01$ & 2.41 & 96.6 & $0.39 \pm 0.02$ & 5.36 & 104.4 \\
\hline & 0.10 & $0.11 \pm 0.003$ & 3.11 & 92.1 & $0.11 \pm 0.01$ & 7.33 & 93.2 \\
\hline
\end{tabular}

Table 4-Accuracy for the Assay of Marker Constituents of SYS.

\begin{tabular}{ccccc}
\hline \hline Compound & Spiked amount $(\mu \mathrm{g})$ & Measured amount $(\mu \mathrm{g})$ & Accuracy $(\%)$ & R.S.D. $(\%)$ \\
\hline \multirow{3}{*}{ paeoniflorin } & 1.31 & $1.35 \pm 0.05$ & 103.5 & 4.64 \\
\cline { 2 - 5 } & 1.06 & $1.14 \pm 0.01$ & 108.1 & 0.71 \\
\cline { 2 - 5 } glycyrrhizin & 0.89 & $0.98 \pm 0.02$ & 111.1 & 1.52 \\
\cline { 2 - 5 } & 1.06 & $1.09 \pm 0.02$ & 102.3 & 2.40 \\
\cline { 2 - 5 } & 0.85 & $0.88 \pm 0.01$ & 102.9 & 0.71 \\
\hline \multirow{2}{*}{ nyyyy}
\end{tabular}

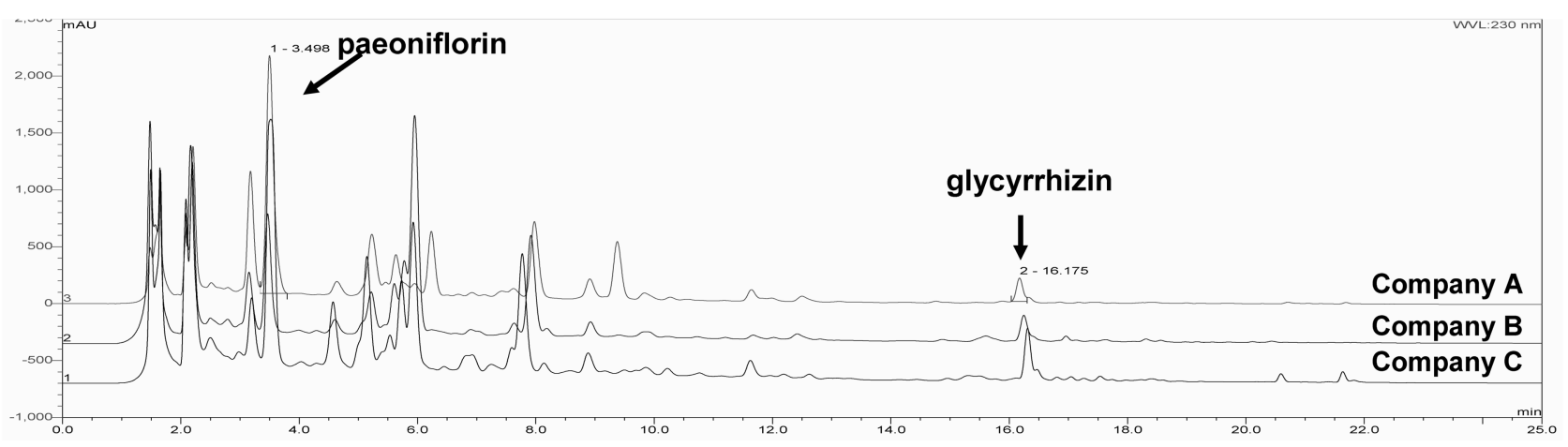

Figure 3-HPLC chromatogram of three commercial SYS products from different companies, A, B and C.

addition. As listed in Table 4, the mean recovery of each compound was $102.3 \sim 111.1 \%$ with R.S.D. values less than $4.6 \%$ $(n=3)$.

\section{Determination of marker compounds in commercial} SYS products

The established method has been applied to the determination of the two marker constituents in commercial SYS granules. Three SYS products by different medicinal companies were used for the determination of each compound. As shown in Figure 3, chromatographic separation of paeoniflorin and glycyrrhizin in each product was well achieved by using the developed method.

J. Kor. Pharm. Sci., Vol. 39, No. 1(2009)

\section{Conclusion}

In this paper, HPLC method for simultaneous determination of the marker constituents of SYS, paeoniflorin and glycyrrhizin, has been developed and validated. The method fulfilled all the requirements to be identified as a reliable and feasible method showing good specificity, precision, linearity and accuracy data. Therefore, this established method is useful for the quality control of SYS by simultaneous quantitative analysis of these constituents.

\section{Acknowledgement}

This research was supported by a grant of the Korea Drug \& 
Food Administration, Republic of Korea (2006).

\section{References}

1) T.H. Xue and R. Roy, Studying traditional Chinese medicine, Science, 300, 740-741 (2003).

2) S.Y. Wang, W. Zheng and GJ. Galletta, Cultural system affects fruit quality and antioxidant capacity in strawberries, J. Agric. Food Chem., 50, 6534-6542 (2002).

3) J.O. Bennett, O. Yu, L.G. Heatherly and H.B. Krishnan, Accumulation of genistein and daidzein, soybean isoflavones implicated in promoting human health, is significantly elevated by irrigation, J. Agric. Food Chem., 52, $7574-7579$ (2004).

4) S.T. Lund and J. Bohlmann, The molecular basis for wine grape quality - a volatile subject, Science, 311, 804-805 (2006).

5) M.J. Antonnen, K.I. Hoppula, R. Nestby, M.J. Verheul and R.O. Karjalainen., Influence of fertilization, mulch color, early forcing, fruit order, planting date, shading, growing environment, and genotype on the contents of selected phenolics in strawberry (Fragaria x ananassa Duch.) fruits, J. Agric. Food Chem., 54, 2614-2620 (2006).

6) Y.X. Sheng, L. Li, Q. Wang, H.Z. Guo and D.A. Guo, Simultaneous determination of gallic acid, albiflorin, paeoniflorin, ferulic acid and benzoic acid in Si-Wu decoction by high-performance liquid chromatography DAD method, J. Pharmaceut. Biomed. Anal., 37, 805-810 (2005).

7) H.J. Zhang and Y.Y. Cheng, An HPLC/MC method for identifying major constituents in the hypocholesterolemic extracts for Chinese medicine formula 'Xue-Fu-Zhu-Yu decoction', Biomed. Chromatogr., 20, 821-826 (2006).
8) Y.B. Huang, P.C. Wu, C.S. Su, Y.C. Wu and Y.H. Tsai, Simultaneous quantification of twelve bioactive components in SanHuang-Xie-Xin-Tang by HPLC, Phytochem. Anal., 17, 439446 (2006).

9) C.Y. Li, C.H. Chiu, H.S. Huang, C.H. Lin and T.S. Wu, Highperformance liquid chromatographic method for simultaneous quantification of eight major biologically active ingredients in 'Da-Chai-Hu-Tang' preparation, Biomed. Chromatogr, 20, 305-308 (2006).

10) H.K. Hansen, S.H. Hansen, M. Kraunsoe and G.M. Petersen, Comparison of high-performance liquid chromatography and capillary electrophoresis methods for quantitative determination of glycyrrhizinic acid in pharmaceutical preparations, Eur. J. Pharm. Sci., 9, 41-46 (1999).

11) Y.C. Wang and Y.S. Yang, Simultaneous quantification of flavonoids and triterpenoids in licorice using HPLC, J. Chromatogr. B Analyt. Technol. Biomed. Life Sci., 850, 392-399 (2007).

12) X. Zhao and Y. Sun, Analysis of Paeoniae Radix by high-performance liquid chromatography-electrospray ionization-mass spectrometry, Anal. Sci., 19, 1313-1315 (2003).

13) H. Cui, C. He and GW. Zhao, Determination of polyphenols by high-performance liquid chromatography with inhibited chemiluminescence detection, J. Chromatogr. A, 855, 171-179 (1999).

14) A. Escarpa and M.C. Gonzalez, Fast separation of (poly)phenolic compounds from apples and pears by high-performance liquid chromatography with diode-array detection, J. Chromatogr. A, 830, 301-309 (1999). 